\title{
Public prescription drug plan coverage for antiretrovirals and the potential cost to people living with HIV in Canada: a descriptive study
}

\author{
Deborah Yoong BScPhm PharmD, Ahmed M. Bayoumi MD MSc, Linda Robinson BScPhm, \\ Beth Rachlis PhD, Tony Antoniou PharmD PhD
}

Abstract

Background: Antiretrovirals are expensive and people living with HIV may experience a range of financial burdens when accessing these medications. Our aim was to describe the policy of all Canadian public drug insurance programs for antiretroviral drugs and illustrated how these policies might affect patients' annual out-of-pocket expenditures.

Methods: In December 2017, we reviewed public drug programs offering antiretroviral coverage in Canada using government websites to summarize eligibility criteria. We estimated the annual out-of-pocket costs incurred by people living with HIV by applying the cost-sharing rules to 2 hypothetical cases, a single man and a married woman with a net household income of $\$ 39000$ and $\$ 80000$, respectively, receiving identical prescriptions in different jurisdictions.

Results: We observed substantial variation in the subsidy provided based mainly on geography, income and age. All 5 federal programs and 6 of 13 provincial and territorial jurisdictions offered universal coverage. In the remaining regions, patients spend up to several thousand dollars annually depending on income (Manitoba), age and income (Ontario, Saskatchewan) and age, income and drug costs (Quebec and Newfoundland and Labrador). We found the greatest variation for our higher income case, with out-of-pocket expenses ranging from 0 to over $50 \%$ of the antiretroviral cost.

Interpretation: There is considerable inter- and intra-jurisdiction heterogeneity in the cost-sharing policies for antiretrovirals across Canada's public drug programs. Policy reforms that either eliminate or set national standards for copayments, deductibles or premiums would minimize variation and could reduce the risk of cost-associated non-adherence to HIV therapy.

\footnotetext{
nternational guidelines recommend that combination antiretroviral therapy for people living with human immunodeficiency virus (HIV) infection be initiated promptly after diagnosis and continued for life to decrease morbidity, mortality and the risk of transmission to uninfected people. ${ }^{1-5}$ However, in Canada, the list price for firstline single-tablet regimens is approximately $\$ 15000$ per year, ${ }^{6}$ and none of Canada's publicly funded drug plans provide universal coverage of all prescription drugs. In the absence of a single national plan, each of Canada's 10 provinces and 3 territorial governments manages and delivers health care services for its residents, including medication coverage (several federal plans insure specific populations). Each plan decides on the eligibility criteria for public drug insurance and the level of subsidy and selects the products to be listed on its drug formulary. Consequently, Canadians with identical prescriptions may pay substantially different amounts and may rely on private insurance, public funders, out-of-pocket payments or a combination of these to pay for their medication. ${ }^{7-9}$
}

Financial burdens are associated with medication nonadherence. ${ }^{10}$ Research indicates that medicine costs sometimes compete with other demands, leading to cost-related nonadherence: patients may forego prescribed medications in favour of spending their resources on other priorities or they may alter a medication's dosing to make a prescription last longer. ${ }^{10,11}$ Accordingly, it may be important to address cost-sharing mechanisms that require large out-of-pocket payments for antiretrovirals, particularly for people without drug insurance and those with more limited income. ${ }^{12,13}$ Nonadherence to antiretroviral therapy can lead to uncontrolled HIV replication and subsequently

Competing interests: Ahmed Bayoumi reports receiving personal fees from the Canadian Agency for Drugs and Technologies in Health, outside the submitted work. No other competing interests were declared.

This article has been peer reviewed.

Correspondence to: Deborah Yoong, yoongd@smh.ca

CMAJ Open 2018. DOI:10.9778/cmajo.20180058 
to increased risks of disease progression, ${ }^{14}$ drug resistance ${ }^{15}$ and HIV transmission. ${ }^{16,17}$ Because viral suppression prevents infection at the individual level ${ }^{5,16-18}$ and may be effective in reducing transmission at the population level, ${ }^{19-21}$ consideration should be given to providing affordable and accessible antiretroviral therapy for all; this has been highlighted as a fundamental component of a public response to the HIV epidemic. ${ }^{2}$ Our primary objective was to describe and compare the reimbursement policy of all Canadian public drug insurance programs for antiretroviral drugs. To illustrate the financial burden that people living with $\mathrm{HIV}$ in Canada encounter in trying to obtain their HIV medication, we used 2 clinical scenarios to estimate the potential annual out-of-pocket expenditures when patients are prescribed HIV medication in each jurisdiction.

\section{Methods}

\section{Sources of data}

We searched the government websites of all jurisdictions in Canada that offered coverage for antiretroviral drugs in December 2017 (Appendix 1, available at www.cmajopen.ca/content/6/4/ E551/suppl/DC1). We extracted data including eligibility criteria, cost-sharing rules, permission to coordinate with private payers, and whether there were restrictions pertaining to the prescriber or the dispensing pharmacy (Appendix 2, available at www.cmajopen.ca/content/6/4/E551/suppl/DC1). To validate the data, we asked a pharmacist in each jurisdiction who had expertise in providing HIV care or who was familiar with that jurisdiction's antiretroviral reimbursement plans and the systems required to secure drug coverage for individual patients to review the information for accuracy (with the exception of the federal programs and Nunavut, where we did not have an email address of a representative pharmacist). If there were incorrect data, the pharmacist consulted another local representative and a consensus was reached. We focused exclusively on HIV treatment; we did not assess coverage of antiretrovirals for HIV prevention.

\section{Patient scenarios}

To illustrate and compare the annual out-of-pocket expenditures for antiretrovirals in working people, we created 2 clinical scenarios that reflected typical patients seen in clinical practice who did not have any workplace or private drug insurance and did not belong to groups typically eligible for publicly funded drug coverage, such as seniors (age $\geq 65 \mathrm{yr}$ ), children and recipients of social assistance. The first case was a single man, aged 30 years, with no dependents and a net annual household income of $\$ 39000$. The second case was a married woman, aged 48 years, with 2 children and a net annual household income of $\$ 80000$. In each case, the person was prescribed abacavir/lamivudine/dolutegravir, a commonly prescribed first-line single-tablet antiretroviral regimen listed in all public drug formularies in Canada.

\section{Calculation of costs}

For each jurisdiction, we calculated each person's expected annual expenditure for the antiretroviral regimen according to the applicable plan and the cost-sharing rules (Appendix 3, avail- able at www.cmajopen.ca/content/6/4/E551/suppl/DC1). For example, in Nova Scotia, for both case scenarios, there is no premium and no deductible, but there are 4 copayments of $\$ 11.25$ for each 90-day prescription totalling $\$ 45$ annually. While there is also no premium in Ontario, the man with an income of $\$ 39000$ would be required to pay an annual deductible of $\$ 1344$ (3.4\% of $\$ 39000$ ) plus 4 copayments of $\$ 2$ for each of his prescriptions, resulting in $\$ 1352$ paid out-of-pocket. Each calculation was verified by the same pharmacist who confirmed coverage details and any discrepancies were resolved by consensus with a second local representative. We assumed a 90-day supply was obtained 4 times a year and, to simplify comparisons, we used the same income in each region, although programs use different income values to calculate benefits. For example, deductibles in Ontario are calculated using net household income, while Manitoba calculates a "total adjusted family income" as the total taxable income minus $\$ 3000$ for each dependent under the age of 18 years. We calculated prescription costs using the amount reimbursed by Ontario's Ministry of Health and Long-Term Care to pharmacies, as listed on the Ontario Drug Benefit Formulary in December 2017. ${ }^{6}$ We therefore assumed that prices were similar across jurisdictions and that any effects of negotiated price discounts were minimal and excluded mark-ups and professional fees. This was a descriptive, nonqualitative analysis without predetermined hypotheses; thus, no statistical analyses were conducted.

\section{Ethics approval}

Because this study did not involve human subjects, ethics approval was not required.

\section{Results}

\section{Public prescription plans}

We found inter- and intra-jurisdictional variability in costsharing rules for antiretrovirals across public drug programs (Table 1, Table 2). Five federal drug insurance programs and at least 1 program in each province or territory provided some form of financial assistance for registered residents of Canada requiring HIV treatment. All 5 federal programs, which are portable across the country, fully subsidized the cost of antiretrovirals for eligible patients regardless of their age or income. Beyond these programs, the governments of British Columbia, Alberta, Northwest Territories, Nunavut, and Prince Edward Island also offered universal coverage of antiretrovirals for all of their residents living with HIV. In New Brunswick, because premiums and co-payments were waived and not collected, the plan functioned as a universal one.

All other jurisdictions had either a co-payment or a deductible, or both, for antiretrovirals. Quebec also collected a yearly income-based premium of $\$ 0$ to $\$ 667$ from noninsured people whether they purchased drugs or not. There was no limit to the annual income-based deductible collected in Manitoba, Newfoundland and Labrador, Ontario and Saskatchewan, and a high income did not disqualify a person from receiving government assistance in any jurisdiction except in Newfoundland and Labrador, where there was no 
assistance available for a resident with a net annual household income of more than $\$ 150000$.

In almost all regions where antiretroviral expenses were shared, fees were reduced or waived for people with very low incomes. Seniors, in contrast, incurred the same out-of-pocket expenses as their non-senior counterparts with the same income in Manitoba and Nova Scotia. Ontario, Quebec, Saskatchewan and Newfoundland and Labrador had multiple programs with eligibility criteria varying according to age, income or drug costs.

The programs also differed in other ways. First, although British Columbia, Alberta, New Brunswick and Prince Edward Island provide antiretrovirals at no cost to their residents, these provinces did not provide universal coverage of prescriptions not related to HIV. Second, coordination of benefits with private insurers was allowed in all programs sharing antiretroviral costs except Quebec. Similarly, patients living in New Brunswick were not eligible for free antiretrovirals from the government if they received any benefits from a private plan, whether the plan was full or partial. Third, there were interprovincial differences in antiretroviral prescribing and dispensing, with 7 jurisdictions (Alberta, British Columbia, New Brunswick, Nova Scotia, Ontario, Saskatchewan and Yukon) and Correctional Service Canada placing restrictions on the prescriber authorizing the regimen and 5 provinces (Alberta, British Columbia, New Brunswick, Nova Scotia and Prince Edward Island) and Correctional Service Canada assigning designated pharmacies to dispense these therapies.

\section{Patient scenarios and calculated costs}

The annual cost of single-tablet abacavir/lamivudine/dolutegravir in the Ontario Drug Benefit Formulary was \$15 552. ${ }^{6}$ Our first hypothetical case of a single man with an annual income of $\$ 39000$ had no out-of-pocket expenses if he lived in Alberta, British Columbia, Northwest Territories, Nunavut, New Brunswick or Prince Edward Island, but he had an annual prescription cost that varied from $\$ 45$ to $\$ 1944$ if he resided elsewhere (Figure 1, Appendix 3). Our second hypothetical case, a married woman with about twice the annual household income of the first case, received her medications at no cost in the same 6 regions, incurred the same expense if she lived in Nova Scotia, Yukon and Quebec (although she would have had to pay a higher premium when she filed her higher household tax return) and paid $\$ 2720$ to $\$ 7993$ (17\% to over 50\% of the antiretroviral cost) if she lived in one of the remaining regions (Figures 1 and 2, Appendix 3).

\section{Interpretation}

We studied the reimbursement policies of public drug plans in Canada for antiretrovirals. Our main finding was that there is considerable variability across public drug insurance programs for these high-cost drugs, such that a person may incur hundreds or thousands of dollars of additional costs solely on the basis of where they live. Our descriptive analysis showed that this disparity was most striking for high-income

Table 1: Characteristics of all publicly funded federal drug plans in Canada that cover antiretrovirals for adults and seniors, December 2017

\begin{tabular}{|c|c|c|c|c|c|c|c|}
\hline \multirow[b]{2}{*}{ Public drug program; eligibility } & \multicolumn{4}{|c|}{ Cost-sharing requirements } & \multicolumn{3}{|c|}{ Restrictions } \\
\hline & Premium* & Deductible & $\begin{array}{c}\text { Co- } \\
\text { paymentł }\end{array}$ & $\begin{array}{l}\text { Coordinates } \\
\text { with other } \\
\text { payers }\end{array}$ & Prescriber & Pharmacy & $\begin{array}{l}\text { Plan covers } \\
\text { drugs not } \\
\text { related to HIV }\end{array}$ \\
\hline $\begin{array}{l}\text { Non-Insured Health Benefits Program } \\
\text { Registered First Nations persons, Inuit } \\
\text { recognized by an Inuit land claim organization }\end{array}$ & $\$ 0$ & $\$ 0$ & $\$ 0$ & Private first & None & None & Yes \\
\hline $\begin{array}{l}\text { Interim Federal Health Program } \\
\text { Resettled refugees, protected persons, } \\
\text { refugee claimants, victims of human } \\
\text { trafficking, detainees }\end{array}$ & $\$ 0$ & $\$ 0$ & $\$ 0$ & NA & $\begin{array}{l}\text { Follows } \\
\text { provincial } \\
\text { policy }\end{array}$ & $\begin{array}{l}\text { Follows } \\
\text { provincial } \\
\text { policy }\end{array}$ & $\begin{array}{l}\text { Follows } \\
\text { provincial } \\
\text { formulary }\end{array}$ \\
\hline $\begin{array}{l}\text { Veteran Affairs Canada Prescription Drug } \\
\text { Program } \\
\text { Royal Canadian Mounted Police members, } \\
\text { Canadian war veterans, eligible Canadian Armed } \\
\text { Forces members, certain wartime civilians }\end{array}$ & $\$ 0$ & $\$ 0$ & $\$ 0$ & Private first & None & None & $\begin{array}{l}\text { Benefits } \\
\text { related to plan }\end{array}$ \\
\hline $\begin{array}{l}\text { Correctional Service Canada } \\
\text { Federal inmates }\end{array}$ & $\$ 0$ & $\$ 0$ & $\$ 0$ & NA & $\begin{array}{l}\text { CSC } \\
\text { institution } \\
\text { physician }\end{array}$ & $\begin{array}{c}\text { CSC } \\
\text { institution }\end{array}$ & Yes \\
\hline $\begin{array}{l}\text { Canadian Forces Health Services } \\
\text { Canadian Armed Forces personnel, } \\
\text { authorized visiting military forces, foreign } \\
\text { military exchange personnel and their } \\
\text { dependents }\end{array}$ & $\$ 0$ & $\$ 0$ & $\$ 0$ & NA & None & $\begin{array}{c}\text { Local base } \\
\text { pharmacy } \\
\text { or } \\
\text { community } \\
\text { pharmacy if } \\
\text { after hours }\end{array}$ & Yes \\
\hline \multicolumn{8}{|c|}{$\begin{array}{l}\text { Note: } C S C=\text { Correctional Service Canada, NA = not applicable. } \\
{ }^{*} \text { The amount an individual must pay to be enrolled in the program. } \\
\text { †The amount that must be paid by the individual before the program pays for any part of the drug costs. } \\
\ddagger \text { The amount or portion an individual pays with each prescription filled. }\end{array}$} \\
\hline
\end{tabular}


Table 2 (part 1 of 4): Characteristics of all publicly funded provincial/territorial drug plans in Canada that cover antiretrovirals for adults and seniors, December 2017

\begin{tabular}{|c|c|c|c|c|c|c|c|c|}
\hline \multirow[b]{2}{*}{ Public drug program } & \multirow[b]{2}{*}{ Eligibility } & \multicolumn{4}{|c|}{ Cost-sharing plan } & \multicolumn{2}{|c|}{ Restrictions } & \multirow{2}{*}{$\begin{array}{l}\text { Plan } \\
\text { covers } \\
\text { drugs not } \\
\text { related to } \\
\text { HIV }\end{array}$} \\
\hline & & Premium* & Deductible† & Copayment‡ & $\begin{array}{l}\text { Coordinates } \\
\text { with other } \\
\text { payers }\end{array}$ & Prescriber & Pharmacy & \\
\hline \multicolumn{9}{|l|}{ Alberta } \\
\hline $\begin{array}{l}\text { Alberta Health Care } \\
\text { Insurance Plan, } \\
\text { Specialized High Cost } \\
\text { Drug Program }\end{array}$ & $\begin{array}{l}\text { Registered§ Alberta } \\
\text { residents }\end{array}$ & $\$ 0$ & $\$ 0$ & $\$ 0$ & No & Yes & Yes & No \\
\hline \multicolumn{9}{|l|}{ British Columbia } \\
\hline $\begin{array}{l}\text { British Columbia } \\
\text { Centre for Excellence } \\
\text { HIV Drug Treatment } \\
\text { Program }\end{array}$ & $\begin{array}{l}\text { HIV-positive BC } \\
\text { residents } \\
\text { Residents with } \\
\text { coverage from another } \\
\text { jurisdiction awaiting BC } \\
\text { medical coverage }\end{array}$ & $\$ 0$ & $\$ 0$ & $\$ 0$ & No & Yesף & Yes & No \\
\hline \multicolumn{9}{|l|}{ Manitoba } \\
\hline Pharmacare Program & $\begin{array}{l}\text { Registered Manitoba } \\
\text { residents }\end{array}$ & $\$ 0$ & $\begin{array}{c}3.05 \%-6.9 \% \\
\text { of adjusted } \\
\text { family } \\
\text { income } \\
\text { not prorated; } \\
\text { minimum } \\
\$ 100 / y r\end{array}$ & $\$ 0$ & $\begin{array}{l}\text { Province } \\
\text { first }\end{array}$ & No & No & Yes \\
\hline $\begin{array}{l}\text { Employment and } \\
\text { Income Assistance } \\
\text { Program - } \\
\text { Prescription Drugs }\end{array}$ & & $\$ 0$ & $\$ 0$ & $\$ 0$ & $\begin{array}{l}\text { Province } \\
\text { first }\end{array}$ & No & No & Yes \\
\hline \multicolumn{9}{|l|}{ New Brunswick } \\
\hline $\begin{array}{l}\text { Prescription Drug } \\
\text { Program, HIV/AIDS } \\
\text { Plan }\end{array}$ & $\begin{array}{l}\text { Registered New } \\
\text { Brunswick residents } \\
\text { not receiving private } \\
\text { insurance }\end{array}$ & $\$ 0 \dagger \dagger$ & $\$ 0$ & $\$ 0 † \dagger$ & No & Yes & Yes & No \\
\hline \multicolumn{9}{|c|}{ Newfoundland and Labrador } \\
\hline \multicolumn{9}{|c|}{ Newfoundland and Labrador Prescription Drug Program (for registered residents of Newfoundland and Labrador) } \\
\hline Foundation Plan & $\begin{array}{l}\text { Residents who qualify } \\
\text { for income support } \\
\text { benefits }\end{array}$ & $\$ 0$ & $\$ 0$ & $\$ 0$ & NA & No & No & Yes \\
\hline Access Plan & $\begin{array}{l}\text { Families with children } \\
\text { with net income } \\
\leq \$ 42870 \\
\text { Couples without } \\
\text { children with net } \\
\text { income } \leq \$ 30009 \\
\text { Single individuals with } \\
\text { net income } \leq \$ 27151\end{array}$ & $\$ 0$ & $\$ 0$ & $\begin{array}{l}20 \%-70 \% \text { of } \\
\text { total } \\
\text { prescription } \\
\text { cost } \\
\text { (varies with } \\
\text { income) }\end{array}$ & Private first & No & No & Yes \\
\hline \multirow[t]{3}{*}{ Assurance Plan } & $\begin{array}{l}\text { Residents with eligible } \\
\text { drug costs that exceed } \\
5 \% \text { of net income } \\
<\$ 40000\end{array}$ & $\$ 0$ & $\$ 0$ & $\begin{array}{c}\text { Copayment } \\
\text { rate }= \\
\text { income } \times \\
5 \% / \text { total drug } \\
\text { expenditure }\end{array}$ & Private first & No & No & Yes \\
\hline & $\begin{array}{l}\text { Residents with eligible } \\
\text { drug costs that exceed } \\
7.5 \% \text { of net income } \\
\text { between } \$ 40000 \text { and } \\
\$ 74999\end{array}$ & $\$ 0$ & $\$ 0$ & $\begin{array}{c}\text { Copayment } \\
\text { rate = } \\
\text { income } \times \\
7.5 \% / \text { total } \\
\text { drug } \\
\text { expenditure }\end{array}$ & Private first & No & No & Yes \\
\hline & $\begin{array}{l}\text { Residents with eligible } \\
\text { drug costs that exceed } \\
10 \% \text { of net income } \\
\$ 75000 \text { to }<\$ 149999\end{array}$ & $\$ 0$ & $\$ 0$ & $\begin{array}{l}\text { Copayment } \\
\text { rate }= \\
\text { income } \times \\
10 \% / \text { total } \\
\text { drug } \\
\text { expenditure }\end{array}$ & Private first & No & No & Yes \\
\hline
\end{tabular}


Table 2 (part 2 of 4): Characteristics of all publicly funded provincial/territorial drug plans in Canada that cover antiretrovirals for adults and seniors, December 2017

\begin{tabular}{|c|c|c|c|c|c|c|c|c|}
\hline \multirow[b]{2}{*}{ Public drug program } & \multirow[b]{2}{*}{ Eligibility } & \multicolumn{4}{|c|}{ Cost-sharing plan } & \multicolumn{2}{|c|}{ Restrictions } & \multirow{2}{*}{$\begin{array}{l}\text { Plan } \\
\text { covers } \\
\text { drugs not } \\
\text { related to } \\
\text { HIV }\end{array}$} \\
\hline & & Premium* & Deductible† & Copayment & $\begin{array}{c}\text { Coordinates } \\
\text { with other } \\
\text { payers }\end{array}$ & Prescriber & Pharmacy & \\
\hline \multicolumn{9}{|c|}{ Newfoundland and Labrador cont'd } \\
\hline \multicolumn{9}{|c|}{ Newfoundland and Labrador Prescription Drug Program (for registered residents of Newfoundland and Labrador) cont'd } \\
\hline 65Plus Plan & $\begin{array}{l}\text { Residents } \geq \text { age } 65 \mathrm{yr} \\
\text { who receive old age } \\
\text { security benefits and } \\
\text { guaranteed income } \\
\text { supplement }\end{array}$ & $\$ 0$ & $\$ 0$ & $\begin{array}{c}\text { Maximum } \$ 6 \\
\text { dispensing } \\
\text { fee }\end{array}$ & Private first & No & No & Yes \\
\hline \multicolumn{9}{|c|}{ Northwest Territories (NWT) } \\
\hline $\begin{array}{l}\text { Extended Health } \\
\text { Benefits for Specified } \\
\text { Disease Conditions }\end{array}$ & $\begin{array}{l}\text { Non-Indigenous } \\
\text { registered NWT } \\
\text { residents }\end{array}$ & $\$ 0$ & $\$ 0$ & $\$ 0$ & Private first & No & No & Yes \\
\hline $\begin{array}{l}\text { Métis Health Benefits } \\
\text { Program }\end{array}$ & $\begin{array}{l}\text { For registered } \\
\text { Indigenous Métis who } \\
\text { are residents of NWT }\end{array}$ & $\$ 0$ & $\$ 0$ & $\$ 0$ & Private first & No & No & Yes \\
\hline $\begin{array}{l}\text { Extended Health } \\
\text { Benefits for Seniors } \\
\text { Program }\end{array}$ & $\begin{array}{l}\text { For non-Indigenous } \\
\text { and non-Métis } \\
\text { registered residents of } \\
\text { NWT who are age } \geq 60 \\
\text { yr }\end{array}$ & $\$ 0$ & $\$ 0$ & $\$ 0$ & Private first & No & No & Yes \\
\hline \multicolumn{9}{|l|}{ Nova Scotia } \\
\hline $\begin{array}{l}\text { Nova Scotia } \\
\text { Department of Health } \\
\text { and Wellness - } \\
\text { Provincial High Cost } \\
\text { Drug Program }\end{array}$ & $\begin{array}{l}\text { Registered residents of } \\
\text { Nova Scotia }\end{array}$ & $\$ 0$ & $\$ 0$ & $\begin{array}{l}\$ 11.25 \\
\text { dispensing } \\
\text { fee }\end{array}$ & Private first & Yes & Yes & No \\
\hline \multicolumn{9}{|l|}{ Nunavut } \\
\hline $\begin{array}{l}\text { Extended Health } \\
\text { Benefits Program }\end{array}$ & $\begin{array}{l}\text { Non-Indigenous } \\
\text { registered Nunavut } \\
\text { residents with a } \\
\text { specified condition } \\
\text { Non-Indigenous } \\
\text { residents age } \geq 65 \text { yr } \\
\text { Registered residents } \\
\text { who are not fully } \\
\text { covered by third-party } \\
\text { insurance }\end{array}$ & $\$ 0$ & $\$ 0$ & $\$ 0$ & Private first & No & No & Yes \\
\hline \multicolumn{9}{|l|}{ Ontario } \\
\hline $\begin{array}{l}\text { Ontario Drug Benefit } \\
\text { program }\end{array}$ & $\begin{array}{l}\text { Residents of long-term } \\
\text { care facilities } \\
\text { Ontario residents } \\
\text { enrolled in the Home } \\
\text { Care Program } \\
\text { People enrolled in } \\
\text { Ontario Works for } \\
\text { income support } \\
\text { People enrolled in the } \\
\text { Ontario Disability } \\
\text { Support Program for } \\
\text { income support and } \\
\text { other services }\end{array}$ & $\$ 0$ & $\$ 0$ & $\begin{array}{l}\$ 2 \text { per } \\
\text { prescription }\end{array}$ & Private first & Yes & No & Yes \\
\hline & $\begin{array}{l}\text { Registered residents } \\
\text { age } \\
\geq 65 \mathrm{yr} \text { with net annual } \\
\text { income } \leq \$ 19300 \\
\text { (single) or net annual } \\
\text { income } \leq \$ 32300 \\
\text { (with spouse) }\end{array}$ & $\$ 0$ & $\$ 0$ & $\begin{array}{l}\$ 2 \text { per } \\
\text { prescription }\end{array}$ & $\begin{array}{l}\text { Province } \\
\text { first }\end{array}$ & Yes & No & Yes \\
\hline
\end{tabular}




\begin{tabular}{|c|c|c|c|c|c|c|c|c|}
\hline \multirow[b]{2}{*}{ Public drug program } & \multirow[b]{2}{*}{ Eligibility } & \multicolumn{4}{|c|}{ Cost-sharing plan } & \multicolumn{2}{|c|}{ Restrictions } & \multirow{2}{*}{$\begin{array}{l}\text { Plan } \\
\text { covers } \\
\text { drugs not } \\
\text { related to } \\
\text { HIV }\end{array}$} \\
\hline & & Premium* & Deductible† & Copayment‡ & $\begin{array}{l}\text { Coordinates } \\
\text { with other } \\
\text { payers }\end{array}$ & Prescriber & Pharmacy & \\
\hline \multicolumn{9}{|l|}{ Ontario cont'd } \\
\hline \multirow[t]{2}{*}{$\begin{array}{l}\text { Ontario Drug Benefit } \\
\text { program cont'd }\end{array}$} & $\begin{array}{l}\text { Registered residents } \\
\text { age } \\
\geq 65 \text { yr with annual net } \\
\text { income }>\$ 19300 \\
\text { (single) or }>\$ 32300 \\
\text { (with spouse) }\end{array}$ & $\$ 0$ & $\$ 100$ & $\begin{array}{l}\$ 6.11 \text { per } \\
\text { prescription }\end{array}$ & $\begin{array}{l}\text { Province } \\
\text { first }\end{array}$ & Yes & No & Yes \\
\hline & $\begin{array}{l}\text { Registered Ontario } \\
\text { residents enrolled in } \\
\text { the Trillium Drug } \\
\text { Program who have } \\
\text { high drug costs relative } \\
\text { to their income }\end{array}$ & $\$ 0$ & $\begin{array}{l}\sim 4 \% \text { of net } \\
\text { household } \\
\text { income; } \\
\text { prorated and } \\
\text { payable } \\
\text { quarterly }\end{array}$ & $\begin{array}{c}\$ 2 \text { per } \\
\text { prescription }\end{array}$ & Private first & Yes & No & Yes \\
\hline \multicolumn{9}{|c|}{ Prince Edward Island (PEI) } \\
\hline $\begin{array}{l}\text { PEI Pharmacare, } \\
\text { AIDS/HIV Program }\end{array}$ & $\begin{array}{l}\text { Registered HIV-positive } \\
\text { residents of Prince } \\
\text { Edward Island }\end{array}$ & $\$ 0$ & $\$ 0$ & $\$ 0$ & No & No & Yes & No \\
\hline \multicolumn{9}{|l|}{ Quebec } \\
\hline \multicolumn{9}{|c|}{ Régie de l'assurance maladie du Québec } \\
\hline \multirow[t]{5}{*}{$\begin{array}{l}\text { Régie de l'assurance } \\
\text { maladie du Québec }\end{array}$} & $\begin{array}{l}\text { Recipients of Social } \\
\text { Assistance and Social } \\
\text { Solidarity Program } \\
\\
\text { Single adults, age } \\
18-25 \mathrm{yr} \text {, living with } \\
\text { parents and full-time } \\
\text { students in an } \\
\text { educational institution } \\
\text { at the secondary, } \\
\text { college or university } \\
\text { level }\end{array}$ & $\$ 0$ & $\$ 0$ & $\$ 0$ & No & No & No & Yes \\
\hline & $\begin{array}{l}\text { Registered Quebec } \\
\text { residents age } \geq 65 \mathrm{yr} \\
\text { receiving } 94 \%-100 \% \\
\text { of the guaranteed } \\
\text { income supplement, } \\
\text { without private } \\
\text { insurance }\end{array}$ & $\$ 0$ & $\$ 0$ & $\$ 0$ & No & No & No & Yes \\
\hline & $\begin{array}{l}\text { Registered Quebec } \\
\text { residents age } \geq 65 \mathrm{yr} \\
\text { receiving } 1 \%-93 \% \text { of } \\
\text { the guaranteed income } \\
\text { supplement, without } \\
\text { private insurance }\end{array}$ & $\begin{array}{c}\$ 0-\$ 667 \\
\text { (varies } \\
\text { with net } \\
\text { family } \\
\text { income) }\end{array}$ & $\begin{array}{l}\$ 19.45 \\
\text { monthly }\end{array}$ & $\begin{array}{c}\text { After } \\
\text { deductible, } \\
\text { (total cost of } \\
\mathrm{Rx}-19.45) \\
\times 34.8 \% \\
\text { up to } \\
\text { maximum of } \\
\$ 52.65 / \mathrm{mo} \\
(\$ 632 / \mathrm{yr})\end{array}$ & No & No & No & Yes \\
\hline & $\begin{array}{l}\text { Registered Quebec } \\
\text { residents age } \geq 65 \mathrm{yr} \\
\text { not receiving any } \\
\text { guaranteed income } \\
\text { supplement, without } \\
\text { private insurance }\end{array}$ & $\begin{array}{c}\$ 0-\$ 667 \\
\text { (varies } \\
\text { with net } \\
\text { family } \\
\text { income) }\end{array}$ & $\begin{array}{l}\$ 19.45 \\
\text { monthly }\end{array}$ & $\begin{array}{c}\text { After } \\
\text { deductible, } \\
\text { (total cost of } \\
\mathrm{Rx}-19.45) \\
\times 34.8 \% \text { up } \\
\text { to maximum } \\
\text { of } \$ 88.83 / \mathrm{mo} \\
(\$ 1066 / \mathrm{yr})\end{array}$ & No & No & No & Yes \\
\hline & $\begin{array}{l}\text { Registered residents of } \\
\text { Quebec without private } \\
\text { insurance }\end{array}$ & $\begin{array}{c}\$ 0-\$ 667 \\
\text { (varies } \\
\text { with net } \\
\text { family } \\
\text { income) }\end{array}$ & $\begin{array}{l}\$ 19.45 \\
\text { monthly }\end{array}$ & $\begin{array}{c}\text { After } \\
\text { deductible, } \\
\text { (total cost of } \\
\mathrm{Rx}-19.45 \text { ) } \\
\times 34.5 \% \text { up } \\
\text { to maximum } \\
\text { of } \$ 88.83 / \mathrm{mo} \\
(\$ 1066 / \mathrm{yr})\end{array}$ & No & No & No & Yes \\
\hline
\end{tabular}


Table 2 (part 4 of 4): Characteristics of all publicly funded provincial/territorial drug plans in Canada that cover antiretrovirals for adults and seniors, December 2017

\begin{tabular}{|c|c|c|c|c|c|c|c|c|}
\hline \multirow[b]{2}{*}{ Public drug program } & \multirow[b]{2}{*}{ Eligibility } & \multicolumn{4}{|c|}{ Cost-sharing plan } & \multicolumn{2}{|c|}{ Restrictions } & \multirow{2}{*}{$\begin{array}{c}\text { Plan } \\
\text { covers } \\
\text { drugs not } \\
\text { related to } \\
\text { HIV }\end{array}$} \\
\hline & & Premium* & Deductible† & Copaymentł & $\begin{array}{l}\text { Coordinates } \\
\text { with other } \\
\text { payers }\end{array}$ & Prescriber & Pharmacy & \\
\hline \multicolumn{9}{|l|}{ Saskatchewan¥¥ } \\
\hline \multicolumn{9}{|c|}{ Saskatchewan Drug Plan } \\
\hline $\begin{array}{l}\text { Special Support } \\
\text { Plan }\end{array}$ & $\begin{array}{l}\text { Registered residents with } \\
\text { drug costs greater than } \\
3.4 \% \text { of taxable income }\end{array}$ & $\$ 0$ & $\begin{array}{l}3.4 \% \text { of } \\
\text { taxable } \\
\text { income }\end{array}$ & $\$ 0$ & $\begin{array}{l}\text { Province } \\
\text { first }\end{array}$ & Yes & No & Yes \\
\hline Seniors' Drug Plan & $\begin{array}{l}\text { Age } \geq 65 \text { yr with net } \\
\text { income of } \leq \$ 68000\end{array}$ & $\$ 0$ & $\$ 0$ & $\begin{array}{l}\text { Maximum of } \\
\$ 25 \text { per } \\
\text { prescription }\end{array}$ & $\begin{array}{l}\text { Province } \\
\text { first }\end{array}$ & Yes & No & Yes \\
\hline $\begin{array}{l}\text { Supplementary } \\
\text { Health Program }\end{array}$ & & $\$ 0$ & $\$ 0$ & $\begin{array}{c}\$ 2 \text { per } \\
\text { prescription }\end{array}$ & $\begin{array}{l}\text { Province } \\
\text { first }\end{array}$ & Yes & No & Yes \\
\hline \multicolumn{9}{|l|}{ Yukon } \\
\hline $\begin{array}{l}\text { Chronic Disease and } \\
\text { Disability Benefits } \\
\text { Program }\end{array}$ & $\begin{array}{l}\text { Registered Yukon } \\
\text { residents }\end{array}$ & $\$ 0$ & $\begin{array}{c}\text { First } \$ 250 \text { of } \\
\text { eligible costs } \\
\text { per year }(\$ 500 \\
\text { per family) }\end{array}$ & $\$ 0$ & Private first & Yes & No & Yes \\
\hline $\begin{array}{l}\text { Pharmacare and } \\
\text { Extended Health } \\
\text { Benefits Program }\end{array}$ & $\begin{array}{l}\text { Registered residents } \\
\text { age } \geq 65 \mathrm{yr} \text {, or }>60 \mathrm{yr} \\
\text { and married to a Yukon } \\
\text { resident who is } \geq 65 \mathrm{yr}\end{array}$ & $\$ 0$ & $\$ 0$ & $\$ 0$ & Private first & Yes & No & Yes \\
\hline \multicolumn{9}{|c|}{ 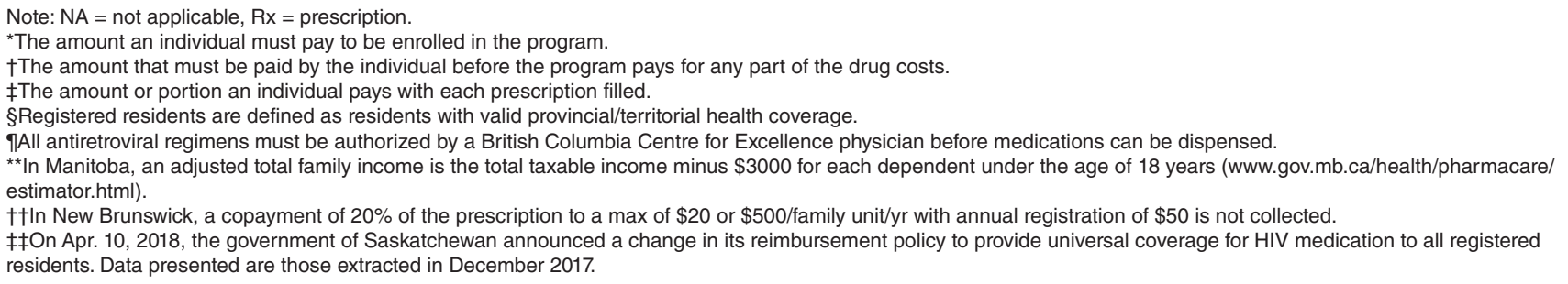 } \\
\hline
\end{tabular}

earners without private drug insurance. For such people, outof-pocket expenses would be $\$ 0$ in 6 regions and could be up to $100 \%$ of the antiretroviral cost in jurisdictions with no maximum annual contribution, namely Saskatchewan, Manitoba, Ontario and Newfoundland and Labrador. While the principle of progressivity - that high-income earners should contribute a greater proportion of their income to shared expenditures - is often cited as a criterion for fairness, our cases illustrated that this philosophy has not been universally adopted across the country. Our case of the lower income man paid a greater proportion of his income for antiretrovirals than our woman with a higher income in Nova Scotia, Quebec and the Yukon (Figure 3). However, even among high-income earners enrolled in cost-sharing plans, our analysis revealed there was disparity in the proportion of income used to calculate medication costs and it varied according to where they lived. A person earning more than $\$ 75000$ would have a deductible of $6.9 \%$ of the family income in Manitoba compared with $4 \%$ in Ontario (Table 2). In addition to variability in government subsidies, our analysis found a considerable range of program complexity in terms of eligibility, administration and restrictions placed on providers or dispensing pharmacies. Although we did not evaluate the administrative overhead costs associated with these complexities, others have indicated that considerable cost savings are possible when drug insurance programs are simplified and streamlined. ${ }^{22}$ The complexity of navigating several programs with differing eligibility criteria and application processes may also prove challenging, especially in specific subgroups of people with HIV, such as people with coexisting cognitive disability or recent immigrants to Canada.

Our finding of heterogeneity in Canada's public drug programs is consistent with prior work; however, the differences across jurisdictions found in other reviews were attributed to the lack of programs for certain subpopulations, ${ }^{8}$ drug costs not exceeding the required premiums or deductibles to receive assistance, ${ }^{7}$ or differences in formulary listing. ${ }^{9}$ In contrast, while we found that all jurisdictions listed most antiretrovirals and, with the exception of Newfoundland and Labrador, each had a program to provide coverage for all subpopulations, the main differences in coverage were due to variations in the amount of individual subsidies based on criteria. In 6 jurisdictions, antiretrovirals were fully covered with an HIV diagnosis being the only requirement for eligibility aside from having provincial/territorial health coverage; in the remaining regions, income 


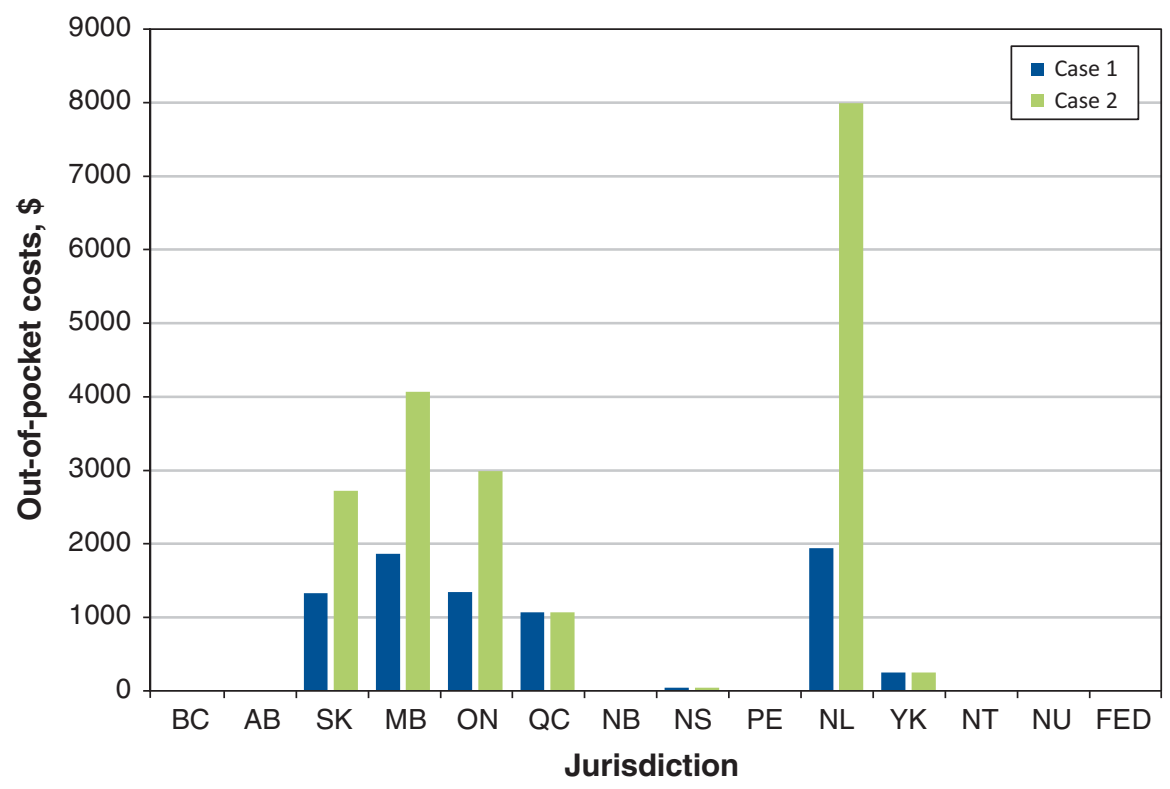

Figure 1: Estimated annual out-of-pocket costs to purchase single-tablet abacavir/lamivudine/dolutegravir in each jurisdiction, excluding mark-ups and professional fees. The total annual cost of the antiretroviral regimen is $\$ 15552$. Case 1 is a single man with no dependents and an annual income of $\$ 39000$. Case 2 is a married woman with 2 dependents and an annual net household income of $\$ 80$ 000. Note: $\mathrm{AB}=$ Alberta, $\mathrm{BC}=$ British Columbia, $\mathrm{FED}=$ federal programs, $\mathrm{MB}=\mathrm{Manitoba}, \mathrm{NB}=\mathrm{New}$ Brunswick, NL = Newfoundland and Labrador, NS = Nova Scotia, NT = Northwest Territories, NU = Nunavut, ON = Ontario, $\mathrm{PE}=$ Prince Edward Island, $\mathrm{QC}=$ Quebec, $\mathrm{SK}=$ Saskatchewan, $\mathrm{YK}=$ Yukon.

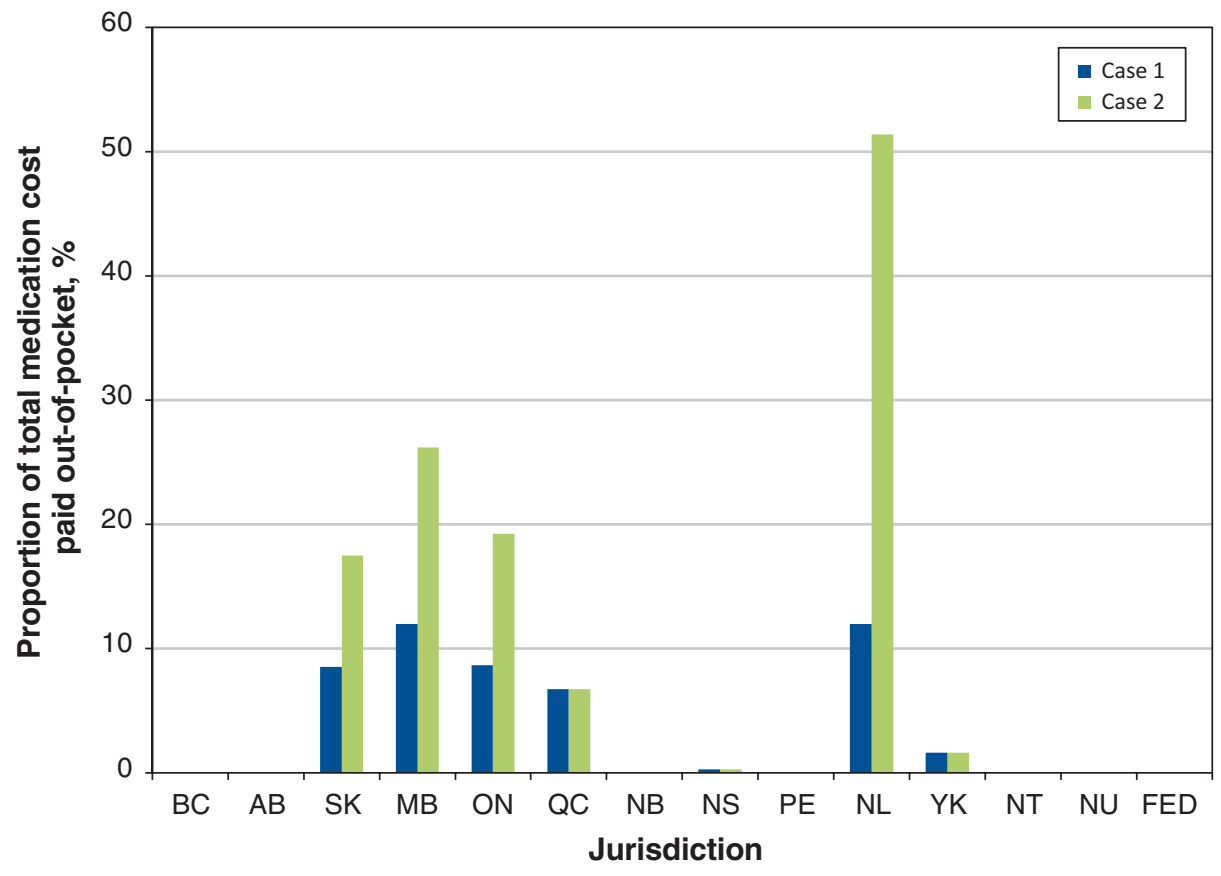

Figure 2: Proportion of annual cost of single-tablet abacavir/lamivudine/dolutegravir paid out-of-pocket. The total annual cost of the antiretroviral regimen is $\$ 15552$. Case 1 is a single man with no dependents and an annual income of $\$ 39000$. Case 2 is a married woman with 2 dependents and an annual net household income of $\$ 80000$. Note: $A B=A$ Alberta, $B C=B r i t i s h$ Columbia, FED $=$ federal programs, $\mathrm{MB}=$ Manitoba, $\mathrm{NB}=$ New Brunswick, $\mathrm{NL}=$ Newfoundland and Labrador, NS $=$ Nova Scotia, NT $=$ Northwest Territories, NU = Nunavut, ON = Ontario, PE = Prince Edward Island, QC = Quebec, SK = Saskatchewan, YK = Yukon. 


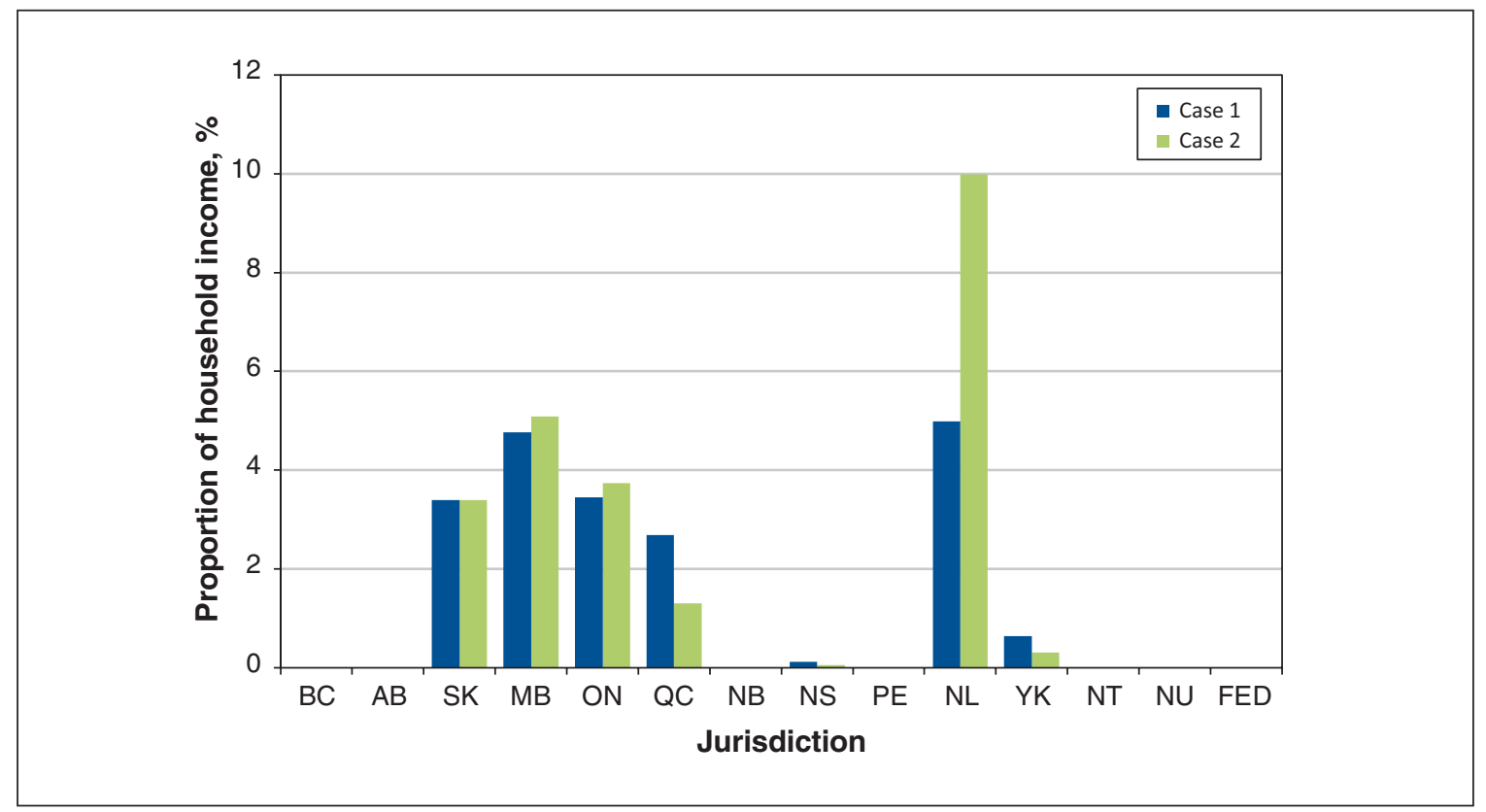

Figure 3: Proportion of household income needed to purchase single-tablet abacavir/lamivudine/dolutegravir in each jurisdiction. The total annual cost of the antiretroviral regimen is $\$ 15552$. Case 1 is a single man with no dependents and an annual income of $\$ 39000$. Case 2 is a married woman with 2 dependents and an annual net household income of $\$ 80000$. Note: $A B=$ Alberta, $\mathrm{BC}=$ British Columbia, FED $=$ federal programs, MB $=$ Manitoba, NB $=$ New Brunswick, NL $=$ Newfoundland and Labrador, NS = Nova Scotia, NT = Northwest Territories, NU = Nunavut, ON = Ontario, PE = Prince Edward Island, QC $=$ Quebec, SK = Saskatchewan, $\mathrm{YK}=$ Yukon.

and age primarily determined the subsidy received. Our results suggest that while the establishment of antiretroviral prescribing programs and formulary listings are necessary conditions for access to coverage, they are insufficient to ensure universal equitable access to antiretrovirals.

Examples of policy options that could address these inequities across the country in eligibility criteria and the value of subsidies include a comprehensive pharmacare program ${ }^{22}$ or explicit national standards for listing and reimbursing drugs for all age and income groups, perhaps with particular attention to drugs that have implications for public health. ${ }^{23}$ For example, medications for tuberculosis and many sexually transmitted infections are universally covered in all or almost all jurisdictions, respectively. On Apr. 10, 2018, the government of Saskatchewan joined 11 jurisdictions and announced it also would provide universal HIV drug coverage to its residents. ${ }^{24}$ In recent years, Canadian jurisdictions (with the exception of Quebec) have established common mechanisms to make listing recommendations through the Common Drug Review and to conduct price negotiations through the pan-Canadian Pharmaceutical Alliance. ${ }^{25} \mathrm{We}$ believe it is also necessary to have national standards and processes to ensure fair and equal cost-sharing mechanisms across the country.

\section{Limitations}

Our study has several limitations. First, we evaluated general reimbursement rules but did not address variations in restrictions for specific antiretrovirals. Second, while we gathered data from government websites that inform the public of all available programs, some information may have been missed as details may have been available only in full policy documents. As we chose only 2 illustrative scenarios, our findings should not be interpreted as being comprehensive (representing all scenarios) or representative (representing the most common scenarios), although we believe they will be relevant for many patients. We did not conduct analyses to determine which reimbursement policies were most prohibitive; this was beyond the scope of our research. Although we solely used the listed drug price from the Ontario Drug Benefit Formulary for our comparison of outof-pocket expenditures and other jurisdictions may have negotiated higher or lower medication prices resulting in different out-of-pocket costs, the actual price would have no implication for patients in jurisdictions that used only income-based deductibles (e.g., Manitoba, Ontario and Saskatchewan) or capped out-of-pocket payments (e.g., Quebec, Yukon). Our study was also restricted to public antiretroviral coverage for adults; we did not examine reimbursement policies for children and youth. Finally, while we documented inequities in cost-sharing, we did not examine whether these inequities result in financial hardship or negative health outcomes. Exploratory research has suggested antiretroviralassociated costs compete with other essential needs. ${ }^{26}$ Our group and others have shown that cost-sharing arrangements for antiretrovirals result in substantial numbers of patients who are unable to afford their medications ${ }^{27,28}$ and consequently, nonadherence. ${ }^{12,13}$ 


\section{Conclusion}

Our study revealed stark inter- and intra-jurisdiction differences for antiretroviral coverage, despite the existence of a public drug plan in each jurisdiction. These disparities result in unequal costs for people living in Canada with identical prescriptions, hindering health equalities across the nation. Addressing cost-sharing inequities may be an important strategy for policy-makers to consider to achieve Canada's commitment to the final 2 goals of the UNAIDS strategy, in which $90 \%$ of all people living with HIV know their HIV status, $90 \%$ of all people diagnosed with HIV receive antiretroviral therapy and $90 \%$ of all people receiving $\mathrm{HIV}$ treatment are virally suppressed by $2020 .^{29}$

\section{References}

1. Panel on Antiretroviral Guidelines for Adults and Adolescents. Guidelines for the use of antiretroviral agents in adults and adolescents living with HIV. Rockville (MD): AIDSinfo; (updated 2018 May 30). Available: http://aidsinfo. nih.gov/contentfiles/lvguidelines/AdultandAdolescentGL.pdf (accessed Mar 2018).

2. Consolidated guidelines on the use of antiretroviral drugs for treating and preventing HIV infections: recommendations for a public bealth approach. 2nd ed. World Health Organization, 2016

3. BHIVA guidelines for the treatment of HIV-1-positive adults with antiretroviral therapy 2015 (2016 interim update). London (UK): British HIV Association; 2016. Available: https://www.bhiva.org/hiv-1-treatment-guidelines (accessed Mar 2018).

4. INSIGHT START Study Group; Lundgren JD, Babiker AG, Gordin F, et al. Initiation of antiretroviral therapy in early asymptomatic HIV infection. $N$ Engl 7 Med 2015;373:795-807.

5. Cohen MS, Chen YQ, McCauley M, et al.; HPTN 052 Study Team. Antiretroviral therapy for the prevention of HIV-1 transmission. N Engl 7 Med 2016;375:830-9.

6. Ontario Ministry of Health and Long Term Care. Drugs funded by Ontario Drug Benefit (ODB) Program: e-formulary. Toronto: Queen's Printer for Ontario; (updated 2018 Sept. 30, version 2.5). Available: https://www.formulary .health.gov.on.ca/formulary/ (accessed Dec 2017).

7. Campbell DJT, Manns BJ, Soril LJJ, et al. Comparison of Canadian public medication insurance plans and the impact on out-of-pocket costs [published erratum in CMAJ Open 2018;6:E11]. CMA7 Open 2017;5:E808-13.

8. Demers V, Melo M, Jackevicius C, et al. Comparisons of provincial prescription drug plans and the impact on patients' annual drug expenditures. CMAF 2008;178:405-9.

9. Hasan H, Howard F, Morgan SG, et al. Provincial disparities of growth hormone coverage for young adult survivors of paediatric brain tumors across Canada. Healthe Policy 2014;9:80-96.

10. Law MR, Cheng L, Dhalla IA, et al. The effect of cost on adherence to prescription medications in Canada. CMA7 2012;184:297-302.

11. Law MR, Cheng L, Kolhatkar A, et al. The consequences of patient charges for prescriptions drugs in Canada: a cross-sectional survey. CMAf Open 2018;6:E63-70.

12. Johnston SS, Juday T, Seekins D, et al. Association between prescription cost sharing and adherence to initial combination antiretroviral therapy in commercially insured antiretroviral-naive patients with HIV. 7 Manag Care Pharm 2012;18:129-45.

13. McAllister J, Beardsworth G, Lavie E, et al. Financial stress is associated with reduced treatment adherence in $\mathrm{HIV}$-infected adults in a resource-rich setting. HIV Med 2013;14:120-4.

14. Bangsberg DR, Perry S, Charlebois ED, et al. Non-adherence to highly active antiretroviral therapy predicts progression to AIDS. AIDS 2001;15:1181-3.

15. Bangsberg DR, Kroetz DL, Deeks SG. Adherence-resistance relationships to combination HIV antiretroviral therapy. Curr HIV/AIDS Rep 2007;4:65-72.

16. Garcia PM, Kalish LA, Pitt J, et al. Maternal levels of plasma human immunodeficiency virus type 1 RNA and the risk of perinatal transmission. $N$ Engl 7 Med 1999;341:394-402.

17. Quinn TC, Wawer MJ, Sewankambo N, et al. Viral load and heterosexual transmission of human immunodeficiency virus type 1. Rakai Project Study Group. N Engl 7 Med 2000;342:921-9.

18. Rodger AJ, Cambiano V, Bruun T, et al. Sexual activity without condoms and risk of HIV transmission in serodifferent couples when the HIV-positive partner is using suppressive antiretroviral therapy. FAMA 2016;316:171-81.
19. Fang CT, Hsu HM, Twu SJ, et al.; Division of AIDS and STD, Center for Disease Control, Department of Health, Executive Yuan. Decreased HIV transmission after a policy of providing free access to highly active antiretroviral therapy in Taiwan. 7 Infect Dis 2004;190:879-85.

20. Granich RM, Gilks CF, Dye C, et al. Universal voluntary HIV testing with immediate antiretroviral therapy as a strategy for elimination of HIV transmission: a mathematical model. Lancet 2009;373:48-57.

21. Montaner JS, Lima VD, Harrigan PR, et al. Expansion of HAART coverage is associated with sustained decreases in HIV/AIDS morbidity, mortality and HIV transmission: the "HIV Treatment as Prevention" experience in a Canadian setting. PLoS One 2014;9:e87872.

22. Morgan SG, Martin D, Gagnon MA, et al. Pharmacare 2020: The future of drug coverage in Canada. Vancouver: Pharmaceutical Policy Research Collaboration, University of British Columbia; 2015. Available: www.pharmacare2020. ca (accessed Dec 2017).

23. Morgan SG, Li W, Yau B, et al. Estimated effects of adding universal public coverage of an essential medicine list to existing public drug plans in Canada. CMA7 2017;189:E295-302.

24. Record $\$ 5.36$ billion invested in improving health care [news release]. Government of Saskatchewan; 2018 Apr. 10. Available: www.saskatchewan.ca/government/ news-and-media/2018/april/10/budget-health (accessed Apr 2018).

25. Allen N, Walker SR, Liberti L, et al. Evaluating alignment between Canadian Common Drug Review reimbursement recommendations and provincial drug plan listing decisions: an exploratory study. CMAF Open 2016;4: E674-8.

26. Rachlis B, Light L. Kopansky-Giles, et al. Challenges with affording HIV medications among participants in the Ontario HIV Treatment Network Cohort Study (OCS) [abstract EPHP12.08]. Proceedings of the Twenty-seventh Annual Conference on HIVIAIDS Research (CAHR); 2018 Apr. 26-29; Vancouver. Canadian Association for HIV Research; 2018:234.

27. Yoong D, Naccarato M, Gough K, et al. Use of compassionate supply of antiretroviral drugs to avoid treatment interruptions of delayed treatment initiation among HIV-positive patients living in Ontario: a retrospective review. Healthc Policy 2015;10:64-77.

28. Chan S, Ireland L, Becker M, et al. The need for compassionate supply of antiretroviral medications provided by the Manitoba HIV Program: a retrospective review [abstract EPHP3.01]. Proceedings of the Twenty-fifth Annual Conference on HIV/AIDS Research (CAHR); 2016 May 12-15; Winnipeg. Canadian Association for HIV Research; 2016:139.

29. 90-90-90 - An ambitious treatment target to help end the AIDS epidemic. Geneva: UNAIDS; 2017. Available: www.unaids.org/en/resources/ documents/2017/90-90-90 (accessed Dec 2017).

Affiliations: Department of Pharmacy (Yoong), St. Michael's Hospital, Toronto, Ont.; Centre for Urban Health Solutions (Bayoumi), Li Ka Shing Knowledge Institute, St. Michael's Hospital, Toronto, Ont.; Department of Medicine (Bayoumi), University of Toronto, Toronto, Ont.; Department of Pharmacy (Robinson), Windsor Regional Hospital, Windsor, Ont.; Ontario HIV Treatment Network (Rachlis), Toronto, Ont.; Department of Family and Community Medicine (Antoniou), St. Michael's Hospital and University of Toronto, Toronto, Ont.; Li Ka Shing Knowledge Institute (Antoniou), St. Michael's Hospital, Toronto, Ont.

Contributors: Deborah Yoong and Tony Antoniou conceived the project; all authors contributed to designing the study. Deborah Yoong and Linda Robinson acquired the data and all authors contributed to analyzing and interpreting the data. Deborah Yoong and Ahmed Bayoumi wrote the first draft of the manuscript and all authors critically revised it for important intellectual content. All authors gave final approval of the version to be published and agreed to be accountable for all aspects of the work.

Acknowledgements: The authors gratefully acknowledge the following people for their review of the drug coverage details of their respective jurisdiction: Linda Akagi, Sarah Burgess, Shanna Chan, Bonnie Corish, Josianne Gauthier, Jeff Kapler, Deborah Kelly, Larry Ring, Nancy Sheehan, Michael Stuber and Jodi Symes. Tony Antoniou is supported by a Canadian Institutes for Health Research New Investigator Award and a University of Toronto Department of Family and Community Medicine Clinician Investigator Award.

Supplemental information: For reviewer comments and the original submission of this manuscript, please see www.cmajopen.ca/content/6/4/ E551/suppl/DC1. 